\title{
Responses of Pseudomonas aeruginosa to antimicrobials
}

\author{
Yuji Morita*, Junko Tomida and Yoshiaki Kawamura \\ Department of Microbiology, School of Pharmacy, Aichi Gakuin University, Nagoya, Japan
}

Edited by:

Joshua D. Nosanchuk, Albert Einstein

College of Medicine, USA

\section{Reviewed by:}

Antonio Oliver, Hospital Son Dureta,

Spain

Yoshimi Matsumoto, Osaka

University, Japan

\section{*Correspondence.}

Yuji Morita, Department of

Microbiology, School of Pharmacy,

Aichi Gakuin University, 1-100

Kusumoto, Chikusa, Nagoya, Aichi

464-8650, Japan

e-mail:yujmor@dpc.agu.ac.jp
Infections caused by Pseudomonas aeruginosa often are hard to treat; inappropriate chemotherapy readily selects multidrug-resistant $P$. aeruginosa. This organism can be exposed to a wide range of concentrations of antimicrobials during treatment; learning more about the responses of $P$. aeruginosa to antimicrobials is therefore important. We review here responses of the bacterium $P$. aeruginosa upon exposure to antimicrobials at levels below the inhibitory concentration. Carbapenems (e.g., imipenem) have been shown to induce the formation of thicker and more robust biofilms, while fluoroquinolones (e.g. ciprofloxacin) and aminoglycosides (e.g., tobramycin) have been shown to induce biofilm formation. Ciprofloxacin also has been demonstrated to enhance the frequency of mutation to carbapenem resistance. Conversely, although macrolides (e.g., azithromycin) typically are not effective against $P$. aeruginosa because of the pseudomonal outer-membrane impermeability and efflux, macrolides do lead to a reduction in virulence factor production. Similarly, tetracycline is not very effective against this organism, but is known to induce the type-III secretion system and consequently enhance cytotoxicity of $P$. aeruginosa in vivo. Of special note are the effects of antibacterials and disinfectants on pseudomonal efflux systems. Sub-inhibitory concentrations of protein synthesis inhibitors (aminoglycosides, tetracycline, chloramphenicol, etc.) induce the MexXY multidrug efflux system. This response is known to be mediated by interference with the translation of the leader peptide PA5471.1, with consequent effects on expression of the PA5471 gene product. Additionally, induction of the MexCD-OprJ multidrug efflux system is observed upon exposure to subinhibitory concentrations of disinfectants such as chlorhexidine and benzalkonium. This response is known to be dependent upon the AlgU stress response factor. Altogether, these biological responses of $P$. aeruginosa provide useful clues for the improvement and optimization of chemotherapy in order to appropriately treat pseudomonal infections while minimizing the emergence of resistance.

Keywords: pseudomonas aeruginosa, anti-bacterial agents, stress responses, multidrug efflux systems, biofilms

\section{INTRODUCTION}

Pseudomonas aeruginosa, a motile, non-fermenting Gramnegative bacterium, is an opportunistic pathogen implicated in respiratory infections, urinary tract infections, gastrointestinal infections, keratitis, otitis media, and bacteremia in patients with compromised host defenses [e.g., cancer, burn, HIV, and cystic fibrosis (CF)]. These infections often result in significant morbidity and mortality. In the 21 st century, when the life expectancy of highly susceptible immunocompromised groups has been extended in most countries, $P$. aeruginosa plays an increasingly prominent role in hospital infections.

This organism is a ubiquitous and metabolically versatile microbe that flourishes in many environments. The bacterium grows under both aerobic and anaerobic conditions, and possesses numerous virulence factors that contribute to its pathogenesis (Schurek et al., 2012). Moreover P. aeruginosa possesses an intrinsic resistance to many antimicrobials because of the bacterium's outer-membrane barrier, the presence of multidrug efflux transporters, and endogenous antimicrobial inactivation (Poole, 2011). Although anti-pseudomonas agents (e.g., carbapenems) have been discovered and developed, P. aeruginosa readily acquires resistance to individual agents via chromosomal mutations and lateral gene transfer (Poole, 2011).

Pseudomonas aeruginosa possesses multifactorial mechanisms of responses and resistance to antimicrobials. While antimicrobials were originally developed and used to kill bacteria, recent work reveals that the biological functions of antibiotics are not limited to bactericidal (killing) or bacteriostatic (growth inhibition) effects (Linares et al., 2006; Aminov, 2013). The most likely function of antibiotics in natural ecosystems is in intercellular "signaling," with specific consequences on the collective behavior of the bacterial population (Linares et al., 2006; Aminov, 2013). Improved genetic tools and cutting-edge technologies (e.g., DNA microarrays) have revolutionized our understanding of microbial physiology (Wecke and Mascher, 2011). Here, we summarize and discuss how $P$. aeruginosa responds to various antimicrobials and survives against its competitors.

\section{RESPONSES TO $\beta$-LACTAMS}

$\beta$-lactams bind to cell wall transpeptidases [penicillin binding proteins (PBPs)], blocking an important step in peptidoglycan biosynthesis (Poole, 2004). Penicillins (e.g., ticarcillin, piperacillin), 
cephalosporins (e.g., ceftazidime, cefepime), monobactams (e.g., aztreonam), and carbapenems (e.g., imipenem, meropenem, and doripenem) are commonly used to treat pseudomonal infections. $P$. aeruginosa is intrinsically resistant to most $\beta$-lactams due to the interplay of the inducible $\beta$-lactamase AmpC and the resistance nodulation cell division (RND) multidrug efflux systems (e.g., MexAB-OprM; Masuda et al., 1999). Benzyl-penicillins (e.g., amoxicillin) and narrow-spectrum cephalosporins are labile to hydrolysis and are strong inducers of $\operatorname{ampC}$, leading to antibiotic degradation, whereas ureidopenicillins (e.g., piperacillin) and extended-spectrum cephalosporins are labile but are weak inducers of ampC (Livermore, 1995). The mexABoprM operon is constitutively expressed in wild-type cells under usual laboratory conditions, where the operon contributes to $P$. aeruginosa's intrinsic resistance to most $\beta$-lactams (except for imipenem) and many other antimicrobial agents, including quinolones, tetracycline, chloramphenicol, and macrolides (Morita et al., 2001). Blocking of dacB-encoded non-essential PBP4 determines a highly efficient and complex $\beta$-lactam resistance response, triggering the overproduction of $\mathrm{AmpC}$ and the specific activation of the $\mathrm{Cre} \mathrm{AB}(\mathrm{Blr} \mathrm{AB})$ two-component regulator (Moya et al., 2009).

Carbapenems are an important class of anti-pseudomonal $\beta$ lactams. Carbapenems are strong inducers that are marginally labile (imipenem) or effectively stable (meropenem; Livermore, 1995). Imipenem also has been shown to strongly induce ampC gene expression in biofilms (Bagge et al., 2004). In addition, $P$. aeruginosa biofilms exposed to imipenem exhibit elevated expression of genes coding for alginate biosynthesis, causing thicker and more robust biofilms (Bagge et al., 2004).

Ceftazidime, a PBP3 inhibitor, does not induce ampC gene expression, but is rather a substrate of AmpC. Ceftazidime impacts the transcription of a large number of genes in $P$. aeruginosa, including those encoding the SOS response repressor LexA-like proteins, causing induced mutagenesis and decreasing ciprofloxacin toxicity (Blazquez et al., 2006). In addition, this antimicrobial shows quorum sensing (QS) inhibitory activity, decreasing the production of a range of QS-regulated virulence factors, in contrast to piperacillin, another PBP3 inhibitor (Skindersoe et al., 2008) These results imply that the QS inhibitory activity of ceftazidime likely is not PBP3-dependent (Skindersoe et al., 2008).

\section{RESPONSES TO FLUOROQUINOLONES}

Fluoroquinolones, particularly ciprofloxacin, are commonly used for the treatment of $P$. aeruginosa infections (Poole, 2011). This class of agents interacts with complexes composed of DNA and either of the two target enzymes, DNA gyrase and/or topoisomerase IV. Primary intrinsic resistance of the wild-type P. aeruginosa to fluoroquinolones is due to MexAB-OprM as well as to MexXY-OprM (Morita et al., 2001). The four RND-type multidrug efflux pumps (MexAB-OprM, MexCD-OprJ, MexEFOprN, and MexXY-OprM) are well recognized as significant determinants of fluoroquinolone resistance in lab and clinical isolates (Poole, 2011), although there are several additional chromosomally encoded efflux pumps that are able to recognize fluoroquinolones (e.g., MexHI-OpmD, MexVW-OprM, the NorM ortholog; Morita et al., 1998; Li et al., 2003; Sekiya et al., 2003; He et al., 2004).

The formation of static biofilms increases when $P$. aeruginosa cells are incubated in the presence of sub-inhibitory concentrations of ciprofloxacin, tobramycin, or tetracycline, while no such sub-inhibitory effect is detected with members of other antibiotic classes, such as carbenicillin, chloramphenicol, or polymyxin (Hoffman et al., 2005; Linares et al., 2006). However, in the presence of a sub-inhibitory concentration of ciprofloxacin (but not of tetracycline or tobramycin), $P$. aeruginosa shows a reduction in swimming and swarming, both of which are important systems of bacterial motility and probably related to the pathogenic process in CF patients (Linares et al., 2006).

Transcriptional responses of $P$. aeruginosa to sub-inhibitory and inhibitory concentrations of ciprofloxacin demonstrate the induction or repression of 100 s of genes (Brazas and Hancock, 2005; Cirz et al., 2006; Brazas et al., 2007). Surprisingly, genes for bacteriophage-like pyocins are up-regulated and mediate fluoroquinolone susceptibility (Brazas and Hancock, 2005). At least one-third of up-regulated genes occur in regulons that are likely controlled by LexA-like SOS response repressor proteins in response to inhibitory concentrations of ciprofloxacin, while down-regulated genes appear to involve virtually every facet of cellular metabolism (Cirz et al., 2006; Brazas et al., 2007). The Lon protease modulates SOS response and consequently ciprofloxacin susceptibility (Breidenstein et al., 2012).

The overall pattern of expression of the DNA replication enzymes suggests a shift from canonical DNA replication enzymes to inducible polymerases in response to inhibitory ciprofloxacin concentrations (Cirz et al., 2006). These inhibitory concentrations of ciprofloxacin create selection pressure in favor of mutants with increased ampC expression (Wolter et al., 2007), while sub-inhibitory levels of ciprofloxacin or ofloxacin enhance the frequency of mutation to carbapenem (especially meropenem) resistance (Tanimoto et al., 2008).

\section{RESPONSES TO AMINOGLYCOSIDES}

Aminoglycosides bind to the $30 \mathrm{~S}$ ribosomal subunit and interfere with protein synthesis, causing mistranslation and ultimately cell death without lysis (Davis, 1987). APH(3')-IIb, a chromosomal aphA-encoded aminoglycoside phosphoryltransferase, is likely responsible for the general non-susceptibility of $P$. aeruginosa to kanamycin (Hachler et al., 1996), and $\mathrm{APH}\left(3^{\prime}\right)$-II predominates in clinical isolates resistant to kanamycin (Poole, 2005). Antipseudomonas aminoglycosides (e.g., amikacin, gentamicin, and tobramycin) therefore can be used in the treatment of $P$. aeruginosa infections (Poole, 2005). Aminoglycoside uptake and subsequent action within bacterial cells is a complex process that involves Lipopolysaccharides (LPS) binding and outer-membrane permeation, cytoplasmic membrane traversal driven by membrane potential, and ribosome disruption, leading to the production of membrane-damaging mistranslated polypeptides (Davis, 1987; Krahn et al., 2012). Primary intrinsic and adaptive resistance to aminoglycosides is due to the MexXY multidrug efflux system in laboratory and clinical isolates (Morita et al., 2012b). The antagonism of aminoglycosides by divalent cations $\mathrm{Mg}^{2+}$ and $\mathrm{Ca}^{2+}$ is well documented in $P$. aeruginosa, and occurs via a process 
that requires the MexXY multidrug efflux system (Mao et al., 2001; Morita et al., 2012a). In wild-type $P$. aeruginosa cells, the MexXY efflux system is inducible by sub-inhibitory concentrations of aminoglycoside- and ribosome-targeting antimicrobials (e.g., chloramphenicol and tetracycline), a process shown to be involved in expression of the PA5471 gene product [recently renamed ArmZ, for anti-repressor MexZ (Morita et al., 2006; Hay et al., 2013)] The PA5471 system also is inducible through interference via translation of the gene's leader peptide, PA5471.1 (Morita et al., 2009). Very recently a clinical strain overproducing MexXY was reported also to harbor a 7-bp deletion in the coding sequence of the leader peptide involved in ribosome-dependent, translational attenuation of PA5471 expression (Guénard et al., 2013).

Transcriptomic analyses confirm that aminoglycosides impact the expression of a myriad of genes (Kindrachuk et al., 2011). While prolonged exposure to sub-inhibitory concentrations of tobramycin causes increased levels of expression, predominantly of the mexXY efflux pump genes, the greatest increases in gene expression levels in response to lethal concentrations of tobramycin involve a number of $P$. aeruginosa heat shock genes (e.g., htpG, ibpA, groES, and asrA; Kindrachuk et al., 2011). Under these conditions, the likely intracellular ATP-dependent AsrA protease is noteworthy because of its modest positive impact on aminoglycoside resistance (Kindrachuk etal., 2011). The Lon protease also is inducible by aminoglycosides (Marr et al., 2007).

Sub-inhibitory concentrations of aminoglycosides, especially tobramycin, induce biofilm formation in P. aeruginosa (Hoffman et al., 2005). Notably, the aminoglycoside tobramycin also induces both swimming and swarming of $P$. aeruginosa (Linares et al., 2006). Also induced is the aminoglycoside response regulator (arr) gene, which is predicted to encode an inner membrane phosphodiesterase. The Arr substrate is cyclic di-guanosine monophosphate (c-di-GMP), a bacterial second messenger that regulates cell surface adhesiveness; c-di-GMP is essential for this induction and contributes to biofilm-specific aminoglycoside resistance (Hoffman et al., 2005).

\section{RESPONSES TO POLYMYXINS}

Owing to the increased prevalence of multidrug-resistant $P$. aeruginosa, polymyxin B and colistin (also called polymyxin E), belonging to a family of antimicrobial cyclic oligopeptides, have returned to favor as a last-resort treatment option, although these agents have strong side effects (e.g., nephrotoxicity) with high incidence (Poole, 2011). Very recently, the polymyxin mutant prevention concentrations (MPCs) for P. aeruginosa were shown to be very high ( $\geq 64 \mu \mathrm{g} / \mathrm{ml}$ ), even for susceptible isolates (i.e., with minimum inhibitory concentration (MIC) ranges of $1-2 \mu \mathrm{g} / \mathrm{ml}$; Choi and Ko, 2013). In the MPC studies, mutation to polymyxin resistance apparently can result from a single mutation (Choi and Ko, 2013).

The mechanism of action of polymyxins involves an initial stage of interaction with the lipid A of the LPS, leading to selfpromoted uptake of polymyxins across the membrane, followed by cell death (Zhang et al., 2000; Fernandez et al., 2013). The most common mechanism of resistance to polymyxin has been shown to arise from modification of LPS lipid A with 4-amino-L-arabinose, a process that has been seen both in in vitro-selected mutants and in CF isolates; other unknown mechanisms remain under investigation (Miller et al., 2011; Poole, 2011; Moskowitz et al., 2012). This modification is carried out by the products of the arnBCADTEF-ugd operon, otherwise known as pmrHFIJKLM-ugd (McPhee et al., 2003; Yan et al., 2007). The two-component ParRS regulator leads to the induction of the LPS modification operon in response to sub-inhibitory concentrations of polymyixin $\mathrm{B}$ and colistin (Fernandez et al., 2010).

Approximately $0.5 \%$ of genes showed significantly altered expression upon exposure to sub-inhibitory concentration of colistin (Cummins et al., 2009), a frequency that is no less dramatic than that seen with the other anti-pseudomonas agents (e.g., ceftazidime, ciprofloxacin, and tobramycin) described above. The most striking alterations were up-regulation of the Pseudomonas quinolone signal (PQS) biosynthetic genes such as the pqsABCDE operon, the phenazine biosynthetic operon, and the arn operon (Cummins et al., 2009).

\section{RESPONSES TO THE MAJOR HUMAN CATIONIC HOST DEFENSE PEPTIDE, LL-37}

The major human cationic host defense peptide LL37 [a.k.a. hCAP-18, FALL-39, or cathelicidin antimicrobial peptide (CAMP)], a 37 -amino-acid, $18-\mathrm{kDa}$ peptide, is encoded by the cathelicidin gene (CAMP) and was originally identified in humans (Kosciuczuk et al., 2012). Sub-inhibitory concentration of LL$37(1 / 4-1 / 128$ of the MIC of $64 \mu \mathrm{g} / \mathrm{ml})$ were shown to prevent biofilm formation by decreasing the attachment of $P$. aeruginosa cells, stimulating twitching motility, and influencing two major QS systems (Las and Rhl), leading to the down-regulation of genes essential for biofilm development (Overhage et al., 2008). Similar results were obtained using the bovine neutrophil peptide indolicidin, but no inhibitory effects on biofilm formation were detected using sub-inhibitory concentrations of the mouse peptide CRAMP (67\% identical with LL-37), polymyxin B, or the bovine bactenecin homolog Bac2A (Overhage et al., 2008).

\section{RESPONSES TO MACROLIDES}

Macrolides such as erythromycin and azithromycin are widely used antibiotics that block translation by binding to the $50 \mathrm{~S}$ ribosomal subunit. $P$. aeruginosa cells are intrinsically resistant to macrolides when tested in standard broth culture; for instance, the MIC of erythromycin for $P$. aeruginosa PAO1 is about $512 \mu \mathrm{g} / \mathrm{mL}$ in Mueller-Hinton broth (e.g., Morita et al., 2001; Buyck et al., 2012). Nonetheless, low-dose macrolides such as azithromycin are effective treatments in patients with chronic lung infections (Jaffe etal., 1998; Kudoh etal., 1998). Even at concentrations (e.g., $2 \mu \mathrm{g} / \mathrm{ml}$ of azithromycin) far below the MIC, macrolides inhibit the QS circuitry of P. aeruginsoa strain PAO1, leading to a reduction in virulence factor production (Tateda et al., 2001). Low-dose azithromycin shows bactericidal activity for $P$. aeruginosa biofilms, but selects for $n f x B$ mutants, which overproduce the MexCD-OprJ efflux pump (Mulet et al., 2009). Notably, the AmpC $\beta$-lactamase produced by $n f x B$ mutants is protective in biofilm growth, although over expression of the MexCD-OprJ pump is known to impair P. aeruginosa's intrinsic resistance, which is dependent on the MexXY/MexAB-OprM efflux pump and the AmpC (Mulet et al., 2011). 
Genome-wide approaches have revealed the QS antagonistic activities of azithromycin (e.g., inhibition of QS, reduction of virulence factor production, and strong induction of typeIII secretion systems; Nalca et al., 2006; Skindersoe et al., 2008). This modulation causes decreased expression of the genes encoding the MexAB-OprM efflux pump in P. aeruginosa (Sugimura et al., 2008). Azithromycin inhibits expression of the small RNAs rsm $Y$ and $r s m Z$, a process that depends on the GacA/Rsm signal transduction pathway; this pathway is known to positively control P. aeruginosa QS (Perez-Martinez and Haas, 2011). Both effects of azithromycin on QS (quorum factor-dependent virulence factor production and cell death) require azithromycin to interact with ribosomes (Kohler et al., 2007). The stationary-phase killing of azithromycin is further enhanced by the production of rhamnolipids, which likely facilitate macrolide uptake (Kohler et al., 2007). The mode of action of azithromycin in vivo also is demonstrated through mutations in $23 \mathrm{~S}$ rRNA that confer azithromycin resistance in bacterial isolates of $P$. aeruginosa in chronically infected CF patients (Marvig et al., 2012). The clinical efficacy of macrolides in treating pseudomonal infections can be partially explained by the increased susceptibility of $P$. aeruginosa to these compounds in eukaryotic cell culture media and biological fluids, due to decreased oprM expression and increased outer-membrane permeability (Buyck et al., 2012).

\section{RESPONSES TO TETRACYCLINES AND CHLORAMPHENICOL}

Tetracyclines are bacteriostatic antibiotics based on a hydroanthracene nucleus, which contains four fused rings. The class also includes glycylcyclines (e.g., tigecycline), a group of semisynthetic tetracycline derivatives containing a glycylamido substitution at position 9 (Yao and Moellering, 2011). Tetracyclines enter bacteria by an energy-dependent process and bind reversibly to the $30 \mathrm{~S}$ ribosomal subunit, preventing the attachment of aminoacyl-tRNA to the ribosomal acceptor A-site in the RNA-ribosome complex (Yao and Moellering, 2011). P. aeruginosa is intrinsically resistant to tetracyclines and glycylcyclines due to the MexAB/MexXY efflux systems (Morita et al., 2001; Dean et al., 2003). Sub-inhibitory concentrations of tetracycline and tigecycline induce the MexXY RND efflux system via a mechanism dependent on the ribosomal inhibitor-inducible PA5471 gene product (Dean et al., 2003; Morita et al., 2006).

Tetracycline increases biofilm formation as well as ciprofloxacin and tobramycin, as described above (Linares et al., 2006). Surprisingly, incubation with tetracycline at concentrations $(\sim 1 \mu \mathrm{g} / \mathrm{ml})$ that do not decrease the growth rate of $P$. aeruginosa increases the expression of type-III secretion system (T3SS) genes exs $A$ and exos, and the presence of tetracycline increases the cytotoxicity of P. aeruginosa by nearly 4-fold (Linares et al., 2006). The T3SS is a mechanism by which bacterial pathogens can deliver effectors directly into the cytoplasm of the eukaryotic host cell (Hauser, 2009). Expression of genes forming the T3SS regulon is triggered by ExaA (Hauser, 2009), a transcriptional activator that autoregulates its own expression by a feedback mechanism (Hauser, 2009). ExoS corresponds to a T3SS-secreted toxin (Hauser, 2009).

Chloramphenicol is a bacteriostatic agent that inhibits protein synthesis by binding reversibly to the peptidyltransferase component of the $50 \mathrm{~S}$ ribosomal subunit and preventing the transpeptidation process of peptide chain elongation (Yao and Moellering, 2011). P. aeruginosa is usually intrinsically resistant to chloramphenicol, in part due to the MexAB-OprM efflux system (Morita et al., 2001). In addition, sub-inhibitory concentrations of chloramphenicol induce the MexXY efflux system via a mechanism dependent on the ribosomal inhibitor-inducible PA5471 gene product (Morita et al., 2006). This effect is reminiscent of the induction of the MexEF-OprN efflux system (via the MexT activator) in response to chloramphenicol and nitrosative stress (Fetar et al., 2011). Chloramphenicol is a nitro-aromatic antimicrobial and resembles a nitrosative stress product (Fetar et al., 2011).

\section{RESPONSE TO BIOCIDES}

Pseudomonas aeruginosa also has been reported to contaminate disinfectants (e.g., chlorhexidine, benzalkonium, and triclosan) in hospital or other such environments, thereby compromising the disinfectant's ability to reduce or eliminate bacterial contamination. Chlorhexidine and benzalkonium are a cationic biguanide and a nitrogen-based quaternary ammonium compound, respectively. These biocides function by affecting the cell membrane, resulting in lysis and the loss of cytoplasmic material (Poole, 2002). The RND-type MexCD-OprJ multidrug efflux pump is induced by sub-inhibitory concentrations of disinfectants such as benzalkonium chloride or chlorhexidine (Morita et al., 2003), a process that is dependent upon the $\mathrm{Alg} U$ stress response factor (Fraud et al., 2008). Global transcriptome response to chlorhexidine includes up-regulation of the mexCD-oprJ and oprH-phoPQ operons and down-regulation of genes encoding proteins involved in membrane transport, oxidative phosphorylation, electron transport, and DNA repair (Nde et al., 2009). A P. aeruginosa variant highly adapted to benzalkonium showed increased resistance to fluoroquinolones, owing to mutations in the quinolone resistance-determining region of gyrA and to mutations in genes (mexR and $n f x B$ ) that encode repressors of mexAB-oprM and mexCD-oprJ, respectively (Mc Cay et al., 2010). Development of chlorhexidine-tolerant sub-populations in $P$. aeruginosa biofilms also is dependent on the mexCD-oprJ genes (Chiang et al., 2012).

Triclosan specifically inhibits fatty acid synthesis through inhibition of bacterial enoyl-acyl carrier protein reductase, although $P$. aeruginosa is intrinsically resistant to triclosan due to the structure of the pseudomonal FabV protein (a triclosan-resistant enoyl-acyl carrier protein reductase) and active efflux. This innate resistance stems from at least five RND efflux pumps, including MexAB-OprM (Mima et al., 2007; Zhu et al., 2010). In P. aeruginosa mutant cells lacking the mexAB-oprM genes, sub-inhibitory concentrations of triclosan lead to alterations in the expression of almost half the genome, with $28 \%$ of genes being significantly up-regulated and $16 \%$ being significantly down-regulated (Chuanchuen and Schweizer, 2012). QS-regulating genes are among the most strongly down-regulated, and surprisingly, iron homeostasis is completely blocked in triclosan-exposed cells, thus mimicking conditions with excess iron (Chuanchuen and Schweizer, 2012).

\section{SUMMING UP AND FURTHER PROSPECTS}

The primary biological responses of $P$. aeruginosa to subinhibitory concentrations of antimicrobials are summarized 
Table 1 | Biological responses of $P$. aeruginosa exposed to various antimicrobials at levels below the inhibitory concentrations.

\begin{tabular}{|c|c|c|}
\hline Antimicrobials & Biological responses & References \\
\hline$\beta$-lactams & Induction of the AmpC $\beta$-lactamase(some $\beta$-lactams are inducers, but others are not) & Livermore (1995) \\
\hline \multirow[t]{2}{*}{ Ceftazidime } & Induction of mutagenesis and decreasing ciprofloxacin toxicity & Blazquez et al. (2006) \\
\hline & Inhibition of quorum sensing & Skindersoe et al. (2008) \\
\hline & Induction of SOS response & Brazas and Hancock (2005) \\
\hline & Up-regulation of the bacteriophage-like pyocins & Brazas and Hancock (2005) \\
\hline & Shift from canonical DNA replication enzymes to inducible polymerases & Cirz etal. (2006) \\
\hline & Enhancement of mutation frequency to $\beta$-lactam resistance. & $\begin{array}{l}\text { Wolter et al. (2007), Tanimoto } \\
\text { et al. (2008) }\end{array}$ \\
\hline \multirow{3}{*}{ Aminoglycosides } & Induction of biofilm formation & Hoffman et al. (2005) \\
\hline & Induction of swimming and swarming & Linares et al. (2006) \\
\hline & Induction of the Lon protease & Marr et al. (2007) \\
\hline \multirow[t]{3}{*}{ Macrolides } & Quorum sensing antagonistic activity(reduction in virulence factor) & Tateda et al. (2001) \\
\hline & Induction of the T3SS & Nalca et al. (2006) \\
\hline & Down-regulation of the MexAB-OprM pump & Sugimura etal. (2008) \\
\hline \multirow[t]{2}{*}{ Tetracycline } & Induction of biofilm formation & Linares etal. (2006) \\
\hline & Induction of the T3SS and cytotoxicity & Linares et al. (2006) \\
\hline Chloramphenicol & Induction of the MexEF-OprN efflux pump & Fetar et al. (2011) \\
\hline
\end{tabular}

in Table 1. Interestingly, clinically useful anti-pseudomonas agents (carbapenems, fluoroquinolones, and aminoglycosides) are known to efficiently kill $P$. aeruginosa planktonic cells when used properly, but lead to more severe biofilm development upon exposure at sub-inhibitory concentrations. This pattern means that optimization of anti-pseudomonas chemotherapy is critical; in the absence of such optimization, chemotherapy fails to treat the infection and readily selects multidrug-resistant $P$. aeruginosa. By contrast, macrolides (which are not effective against $P$. aeruginosa planktonic cells because of the planktonic cells' intrinsic resistance) provide activity that is antagonistic to QS, thereby reducing pseudomonal virulence. These findings regarding responses to antimicrobial agents suggest a route toward conquering $P$. aeruginosa infections. For example, macrolides have been shown to augment the in vitro activity of anti-pseudomonas agents against biofilms (Lutz et al., 2012).

We are aware of potential molecular targets for novel antipseudomonas agents, including essential genes (Morita et al., 2010). Novel class anti-pseudomonas agents should be expected to minimize a severe situation in which few agents are effective against these organisms. However, it is a much more difficult task to develop novel class antimicrobials for $P$. aeruginosa because of the presence of low membrane permeability and the RND multidrug efflux pumps. In addition this organism has the ability to adapt to various stresses, including sub-inhibitory antimicrobial exposure, by recruiting antimicrobial resistance mechanisms, notably that of RND efflux systems such as the MexXY system. While many laboratories are currently screening, so far no efflux pump inhibitors have been made available for clinical settings. Screening for novel antibacterial agents, including efflux pump inhibitors, is currently in progress in many laboratories, including our own.

\section{ACKNOWLEDGMENTS}

This work was supported in part by a Grant-in-Aid for Young Scientists (B) (Kakenhi 23790106) from the Japan Society for the Promotion of Science, and by a research grant from the Institute of Pharmaceutical Life Sciences, Aichi Gakuin University.

\section{REFERENCES}

Aminov, R. I. (2013). Biotic acts of antibiotics. Front. Microbiol. 4:241. doi: $10.3389 /$ fmicb.2013.00241 
Bagge, N., Schuster, M., Hentzer, M., Ciofu, O., Givskov, M., Greenberg, E. P., et al. (2004). Pseudomonas aeruginosa biofilms exposed to imipenem exhibit changes in global gene expression and beta-lactamase and alginate production. Antimicrob. Agents Chemother. 48, 1175-1187. doi: 10.1128/AAC.48.4.1175-1187.2004

Blazquez, J., Gomez-Gomez, J. M., Oliver, A., Juan, C., Kapur, V., and Martin, S. (2006). PBP3 inhibition elicits adaptive responses in Pseudomonas aeruginosa. Mol. Microbiol. 62, 84-99. doi: 10.1111/j.1365-2958.2006. 05366.x

Brazas, M. D., Breidenstein, E. B., Overhage, J., and Hancock, R. E. (2007). Role of lon, an ATP-dependent protease homolog, in resistance of Pseudomonas aeruginosa to ciprofloxacin. Antimicrob. Agents Chemother. 51, 4276-4283. doi: 10.1128/AAC.00830-07

Brazas, M. D., and Hancock, R. E. (2005). Ciprofloxacin induction of a susceptibility determinant in Pseudomonas aeruginosa. Antimicrob. Agents Chemother. 49, 3222 3227. doi: 10.1128/AAC.49.8.3222-3227.2005

Breidenstein, E. B., Bains, M., and Hancock, R. E. (2012). Involvement of the lon protease in the SOS response triggered by ciprofloxacin in Pseudomonas aeruginosa PAO1. Antimicrob. Agents Chemother. 56, 2879-2887. doi: 10.1128/AAC. 06014-11

Buyck, J. M., Plesiat, P., Traore, H., Vanderbist, F., Tulkens, P. M., and Van Bambeke, F. (2012). Increased susceptibility of Pseudomonas aeruginosa to macrolides and ketolides in eukaryotic cell culture media and biological fluids due to decreased expression of oprM and increased outer-membrane permeability. Clin. Infect. Dis. 55, 534-542. doi: 10.1093/cid/cis473

Chiang, W. C., Pamp, S. J., Nilsson, M., Givskov, M., and Tolker-Nielsen, T. (2012). The metabolically active subpopulation in Pseudomonas aeruginosa biofilms survives exposure to membrane-targeting antimicrobials via distinct molecular mechanisms. FEMS Immunol. Med. Microbiol. 65, 245-256. doi: 10.1111/j.1574-695X.2012.00929.x

Choi, M. J., and Ko, K. S. (2013). Mutant prevention concentrations of colistin for Acinetobacter baumannii, Pseudomonas aeruginosa and Klebsiella pneumoniae clinical isolates. J. Antimicrob. Chemother. 69, 275-277. doi: 10.1093/jac/ $\mathrm{dkt} 315$

Chuanchuen, R., and Schweizer, H. P. (2012). Global transcriptional responses to triclosan exposure in Pseudomonas aeruginosa. Int. J. Antimicrob. Agents 40, 114-122. doi: 10.1016/j.ijantimicag.2012.04.008

Cirz, R. T., O’Neill, B. M., Hammond, J. A., Head, S. R., and Romesberg, F. E. (2006). Defining the Pseudomonas aeruginosa SOS response and its role in the global response to the antibiotic ciprofloxacin. J. Bacteriol. 188, 7101-7110. doi: 10.1128/JB.00807-06

Cummins, J., Reen, F. J., Baysse, C., Mooij, M. J., and O’Gara, F. (2009). Subinhibitory concentrations of the cationic antimicrobial peptide colistin induce the Pseudomonas quinolone signal in Pseudomonas aeruginosa. Microbiology 155, 2826-2837. doi: 10.1099/mic.0.025643-0

Davis, B. D. (1987). Mechanism of bactericidal action of aminoglycosides. Microbiol. Rev. 51, 341-350.

Dean, C. R., Visalli, M. A., Projan, S. J., Sum, P. E., and Bradford, P. A. (2003). Effluxmediated resistance to tigecycline (GAR-936) in Pseudomonas aeruginosa PAO1. Antimicrob. Agents Chemother. 47, 972-978. doi: 10.1128/AAC.47.3.972-978.2003

Fernandez, L., Alvarez-Ortega, C., Wiegand, I., Olivares, J., Kocincova, D., Lam, J. S., et al. (2013). Characterization of the polymyxin B resistome of Pseudomonas aeruginosa. Antimicrob. Agents Chemother. 57, 110-119. doi: 10.1128/AAC. 01583-12

Fernandez, L., Gooderham, W. J., Bains, M., McPhee, J. B., Wiegand, I., and Hancock, R. E. (2010). Adaptive resistance to the "last hope" antibiotics polymyxin B and colistin in Pseudomonas aeruginosa is mediated by the novel two-component regulatory system ParR-ParS. Antimicrob. Agents Chemother. 54, 3372-3382. doi: 10.1128/aac.00242-210

Fetar, H., Gilmour, C., Klinoski, R., Daigle, D. M., Dean, C. R., and Poole, K. (2011). mexEF-oprN multidrug efflux operon of Pseudomonas aeruginosa: regulation by the MexT activator in response to nitrosative stress and chloramphenicol. Antimicrob. Agents Chemother. 55, 508-514. doi: 10.1128/AAC. 00830-10

Fraud, S., Campigotto, A. J., Chen, Z., and Poole, K. (2008). MexCD-OprJ multidrug efflux system of Pseudomonas aeruginosa: involvement in chlorhexidine resistance and induction by membrane-damaging agents dependent upon the $\mathrm{AlgU}$ stress response sigma factor. Antimicrob. Agents Chemother. 52, 4478-4482. doi: 10.1128/AAC.01072-08
Guénard, S., Muller, C., Monlezun, L., Benas, P., Broutin, I., Jeannot, K., et al. (2013). Multiple mutations lead to MexXY/OprM-dependent aminoglycoside resistance in clinical strains of Pseudomonas aeruginosa. Antimicrob. Agents Chemother. 58, 221-228. doi: 10.1128/AAC.01252-13

Hachler, H., Santanam, P., and Kayser, F. H. (1996). Sequence and characterization of a novel chromosomal aminoglycoside phosphotransferase gene, aph (')-IIb, in Pseudomonas aeruginosa. Antimicrob. Agents Chemother. 40, 1254-1256.

Hauser, A. R. (2009). The type III secretion system of Pseudomonas aeruginosa: infection by injection. Nat. Rev. Microbiol. 7, 654-665. doi: 10.1038/nrmicro2199 Hay, T., Fraud, S., Lau, C. H., Gilmour, C., and Poole, K. (2013). Antibiotic inducibility of the mexXY multidrug efflux operon of Pseudomonas aeruginosa: involvement of the MexZ anti-repressor ArmZ. PLoS ONE 8:e56858. doi: 10.1371/journal.pone.0056858

He, G. X., Kuroda, T., Mima, T., Morita, Y., Mizushima, T., and Tsuchiya, T. (2004). An $\mathrm{H}(+)$-coupled multidrug efflux pump, PmpM, a member of the MATE family of transporters, from Pseudomonas aeruginosa. J. Bacteriol. 186, 262-265. doi: 10.1128/JB.186.1.262-265.2004

Hoffman, L. R., D’Argenio, D. A., MacCoss, M. J., Zhang, Z., Jones, R. A., and Miller, S. I. (2005). Aminoglycoside antibiotics induce bacterial biofilm formation. Nature 436, 1171-1175. doi: 10.1038/nature03912

Jaffe, A., Francis, J., Rosenthal, M., and Bush, A. (1998). Long-term azithromycin may improve lung function in children with cystic fibrosis. Lancet 351, 420. doi: 10.1016/S0140-6736(05)78360-4

Kindrachuk, K. N., Fernandez, L., Bains, M., and Hancock, R. E. (2011). Involvement of an ATP-dependent protease, PA0779/AsrA, in inducing heat shock in response to tobramycin in Pseudomonas aeruginosa. Antimicrob. Agents Chemother. 55, 1874-1882. doi: 10.1128/AAC.00935-10

Kohler, T., Dumas, J. L., and Van Delden, C. (2007). Ribosome protection prevents azithromycin-mediated quorum-sensing modulation and stationary-phase killing of Pseudomonas aeruginosa. Antimicrob. Agents Chemother. 51, 4243-4248. doi: 10.1128/AAC.00613-07

Kosciuczuk, E. M., Lisowski, P., Jarczak, J., Strzalkowska, N., Jozwik, A., Horbanczuk, J., etal. (2012). Cathelicidins: family of antimicrobial peptides. A review. Mol. Biol. Rep. 39, 10957-10970. doi: 10.1007/s11033-0121997-x

Krahn, T., Gilmour, C., Tilak, J., Fraud, S., Kerr, N., Lau, C. H., et al. (2012). Determinants of intrinsic aminoglycoside resistance in Pseudomonas aeruginosa. Antimicrob. Agents Chemother. 56, 5591-5602. doi: 10.1128/AAC.01446-12

Kudoh, S., Azuma, A., Yamamoto, M., Izumi, T., and Ando, M. (1998). Improvement of survival in patients with diffuse panbronchiolitis treated with low-dose erythromycin. Am. J. Respir. Crit. Care Med. 157, 1829-1832. doi: 10.1164/ajrccm.157.6.9710075

Li, Y., Mima, T., Komori, Y., Morita, Y., Kuroda, T., Mizushima, T., et al. (2003). A new member of the tripartite multidrug efflux pumps, MexVWOprM, in Pseudomonas aeruginosa. J. Antimicrob. Chemother. 52, 572-575. doi: $10.1093 / \mathrm{jac} / \mathrm{dkg} 390$

Linares, J. F., Gustafsson, I., Baquero, F., and Martinez, J. L. (2006). Antibiotics as intermicrobial signaling agents instead of weapons. Proc. Natl. Acad. Sci. U.S.A. 103, 19484-19489. doi: 10.1073/pnas.0608949103

Livermore, D. M. (1995). beta-Lactamases in laboratory and clinical resistance. Clin. Microbiol. Rev. 8, 557-584.

Lutz, L., Pereira, D. C., Paiva, R. M., Zavascki, A. P., and Barth, A. L. (2012). Macrolides decrease the minimal inhibitory concentration of anti-pseudomonal agents against Pseudomonas aeruginosa from cystic fibrosis patients in biofilm. BMC Microbiol. 12:196. doi: 10.1186/1471-2180-12-196

Mao, W., Warren, M. S., Lee, A., Mistry, A., and Lomovskaya, O. (2001). MexXYOprM efflux pump is required for antagonism of aminoglycosides by divalent cations in Pseudomonas aeruginosa. Antimicrob. Agents Chemother. 45, 20012007. doi: 10.1128/AAC.45.7.2001-2007.2001

Marr, A. K., Overhage, J., Bains, M., and Hancock, R. E. (2007). The Lon protease of Pseudomonas aeruginosa is induced by aminoglycosides and is involved in biofilm formation and motility. Microbiology 153, 474-482. doi: 10.1099/mic.0.2006/002519-0

Marvig, R. L., Sondergaard, M. S., Damkiaer, S., Hoiby, N., Johansen, H. K., Molin, S., et al. (2012). Mutations in 23S rRNA confer resistance against azithromycin in Pseudomonas aeruginosa. Antimicrob. Agents Chemother. 56, 4519-4521. doi: 10.1128/AAC.00630-12 
Masuda, N., Gotoh, N., Ishii, C., Sakagawa, E., Ohya, S., and Nishino, T. (1999). Interplay between chromosomal beta-lactamase and the MexAB-OprM efflux system in intrinsic resistance to beta-lactams in Pseudomonas aeruginosa. Antimicrob. Agents Chemother. 43, 400-402.

Mc Cay, P. H., Ocampo-Sosa, A. A., and Fleming, G. T. (2010). Effect of subinhibitory concentrations of benzalkonium chloride on the competitiveness of Pseudomonas aeruginosa grown in continuous culture. Microbiology 156, 30-38. doi: 10.1099/mic.0.029751-0

McPhee, J. B., Lewenza, S., and Hancock, R. E. (2003). Cationic antimicrobial peptides activate a two-component regulatory system, PmrA-PmrB, that regulates resistance to polymyxin B and cationic antimicrobial peptides in Pseudomonas aeruginosa. Mol. Microbiol. 50, 205-217. doi: 10.1046/j.1365-2958.2003. 03673.x

Miller, A. K., Brannon, M. K., Stevens, L., Johansen, H. K., Selgrade, S. E., Miller, S. I., et al. (2011). PhoQ mutations promote lipid A modification and polymyxin resistance of Pseudomonas aeruginosa found in colistin-treated cystic fibrosis patients. Antimicrob. Agents Chemother. 55, 5761-5769. doi: 10.1128/AAC. 05391-11

Mima, T., Joshi, S., Gomez-Escalada, M., and Schweizer, H. P. (2007). Identification and characterization of TriABC-OpmH, a triclosan efflux pump of Pseudomonas aeruginosa requiring two membrane fusion proteins. J. Bacteriol. 189, 7600-7609. doi: 10.1128/JB.00850-07

Morita, Y., Gilmour, C., Metcalf, D., and Poole, K. (2009). Translational control of the antibiotic inducibility of the PA5471 gene required for mexXY multidrug efflux gene expression in Pseudomonas aeruginosa. J. Bacteriol. 191, 4966-4975. doi: 10.1128/JB.00073-09

Morita, Y., Kimura, N., Mima, T., Mizushima, T., and Tsuchiya, T. (2001). Roles of MexXY- and MexAB-multidrug efflux pumps in intrinsic multidrug resistance of Pseudomonas aeruginosa PAO1. J. Gen. Appl. Microbiol. 47, 27-32. doi: 10.2323/jgam.47.27

Morita, Y., Kodama, K., Shiota, S., Mine, T., Kataoka, A., Mizushima, T., et al. (1998). NorM, a putative multidrug efflux protein, of Vibrio parahaemolyticus and its homolog in Escherichia coli. Antimicrob. Agents Chemother. 42, 1778-1782.

Morita, Y., Murata, T., Mima, T., Shiota, S., Kuroda, T., Mizushima, T., et al. (2003). Induction of mexCD-oprJ operon for a multidrug efflux pump by disinfectants in wild-type Pseudomonas aeruginosa PAO1. J. Antimicrob. Chemother. 51, 991-994. doi: 10.1093/jac/dkg173

Morita, Y., Narita, S., Tomida, J., Tokuda, H., and Kawamura, Y. (2010). Application of an inducible system to engineer unmarked conditional mutants of essential genes of Pseudomonas aeruginosa. J. Microbiol. Methods 82, 205-213. doi: 10.1016/j.mimet.2010.06.001

Morita, Y., Sobel, M. L., and Poole, K. (2006). Antibiotic inducibility of the MexXY multidrug efflux system of Pseudomonas aeruginosa: involvement of the antibiotic-inducible PA5471 gene product. J. Bacteriol. 188, 1847-1855. doi: 10.1128/JB.188.5.1847-1855.2006

Morita, Y., Tomida, J., and Kawamura, Y. (2012a). MexXY multidrug efflux system of Pseudomonas aeruginosa. Front. Microbiol. 3:408. doi: 10.3389/fmicb.2012.00408

Morita, Y., Tomida, J., and Kawamura, Y. (2012b). Primary mechanisms mediating aminoglycoside resistance in the multidrug-resistant Pseudomonas aeruginosa clinical isolate PA7. Microbiology 158, 1071-1083. doi: 10.1099/mic.0. 054320-0

Moskowitz, S. M., Brannon, M. K., Dasgupta, N., Pier, M., Sgambati, N., Miller, A. K., et al. (2012). PmrB mutations promote polymyxin resistance of Pseudomonas aeruginosa isolated from colistin-treated cystic fibrosis patients. Antimicrob. Agents Chemother. 56, 1019-1030. doi: 10.1128/AAC.05829-11

Moya, B., Dotsch, A., Juan, C., Blazquez, J., Zamorano, L., Haussler, S., et al. (2009). Beta-lactam resistance response triggered by inactivation of a nonessential penicillin-binding protein. PLoS Pathog. 5:e1000353. doi: 10.1371/journal.ppat. 1000353

Mulet, X., Macia, M. D., Mena, A., Juan, C., Perez, J. L., and Oliver, A. (2009). Azithromycin in Pseudomonas aeruginosa biofilms: bactericidal activity and selection of nfxB mutants. Antimicrob. Agents Chemother. 53, 1552-1560. doi: 10.1128/AAC.01264-08

Mulet, X., Moya, B., Juan, C., Macia, M. D., Perez, J. L., Blazquez, J., et al. (2011). Antagonistic interactions of Pseudomonas aeruginosa antibiotic resistance mechanisms in planktonic but not biofilm growth. Antimicrob. Agents Chemother. 55, 4560-4568. doi: 10.1128/AAC.00519-11
Nalca, Y., Jansch, L., Bredenbruch, F., Geffers, R., Buer, J., and Haussler, S. (2006). Quorum-sensing antagonistic activities of azithromycin in Pseudomonas aeruginosa PAO1: a global approach. Antimicrob. Agents Chemother. 50, 1680-1688. doi: 10.1128/AAC.50.5.1680-1688.2006

Nde, C. W., Jang, H. J., Toghrol, F., and Bentley, W. E. (2009). Global transcriptomic response of Pseudomonas aeruginosa to chlorhexidine diacetate. Environ. Sci. Technol. 43, 8406-8415. doi: 10.1021/es9015475

Overhage, J., Campisano, A., Bains, M., Torfs, E. C., Rehm, B. H., and Hancock, R. E. (2008). Human host defense peptide LL-37 prevents bacterial biofilm formation. Infect. Immun. 76, 4176-4182. doi: 10.1128/IAI. 00318-08

Perez-Martinez, I., and Haas, D. (2011). Azithromycin inhibits expression of the GacA-dependent small RNAs RsmY and RsmZ in Pseudomonas aeruginosa. Antimicrob. Agents Chemother. 55, 3399-3405. doi: 10.1128/AAC. 01801-10

Poole, K. (2002). Mechanisms of bacterial biocide and antibiotic resistance. Symp. Ser. Soc. Appl. Microbiol. 55S-64S. doi: 10.1046/j.1365-2672.92.5s1.8.x

Poole, K. (2004). Resistance to beta-lactam antibiotics. Cell. Mol. Life Sci. 61, 2200 2223. doi: 10.1007/s00018-004-4060-9

Poole, K. (2005). Aminoglycoside resistance in Pseudomonas aeruginosa. Antimicrob. Agents Chemother. 49, 479-487. doi: 10.1128/AAC.49.2.479-487. 2005

Poole, K. (2011). Pseudomonas aeruginosa: resistance to the max. Front. Microbiol. 2:65. doi: 10.3389/fmicb.2011.00065

Schurek, K. N., Breidenstein, E. B. M., and Hancock, R. E. W. (2012). "Pseudomonas aeruginosa: a persistent pathogen in cystic fibrosis and hospital-associated infections," in Antibiotic Discovery and Development, Vol. 1, eds T. J. Dougherty and M. J. Pucci (New York: Springer), 679-715.

Sekiya, H., Mima, T., Morita, Y., Kuroda, T., Mizushima, T., and Tsuchiya, T. (2003). Functional cloning and characterization of a multidrug efflux pump, mexHI-opmD, from a Pseudomonas aeruginosa mutant. Antimicrob. Agents Chemother. 47, 2990-2992. doi: 10.1128/AAC.47.9.2990-2992. 2003

Skindersoe, M. E., Alhede, M., Phipps, R., Yang, L., Jensen, P. O., Rasmussen, T. B., et al. (2008). Effects of antibiotics on quorum sensing in Pseudomonas aeruginosa. Antimicrob. Agents Chemother. 52, 3648-3663. doi: 10.1128/AAC.01230-07

Sugimura, M., Maseda, H., Hanaki, H., and Nakae, T. (2008). Macrolide antibioticmediated down regulation of MexAB-OprM efflux pump expression in $\mathrm{Pseu}$ domonas aeruginosa. Antimicrob. Agents Chemother. 52, 4141-4144. doi: 10.1128/ AAC.00511-08

Tanimoto, K., Tomita, H., Fujimoto, S., Okuzumi, K., and Ike, Y. (2008). Fluoroquinolone enhances the mutation frequency for meropenem-selected carbapenem resistance in Pseudomonas aeruginosa, but use of the high-potency drug doripenem inhibits mutant formation. Antimicrob. Agents Chemother. 52, 3795-3800. doi: 10.1128/AAC.00464-08

Tateda, K., Comte, R., Pechere, J. C., Kohler, T., Yamaguchi, K., and Van Delden, C. (2001). Azithromycin inhibits quorum sensing in Pseudomonas aeruginosa. Antimicrob. Agents Chemother. 45, 1930-1933. doi: 10.1128/AAC.45.6.19301933.2001

Wecke, T., and Mascher, T. (2011). Antibiotic research in the age of omics: from expression profiles to interspecies communication. J. Antimicrob. Chemother. 66, 2689-2704. doi: 10.1093/jac/dkr373

Wolter, D. J., Schmidtke, A. J., Hanson, N. D., and Lister, P. D. (2007). Increased expression of ampC in Pseudomonas aeruginosa mutants selected with ciprofloxacin. Antimicrob. Agents Chemother. 51, 2997-3000. doi: 10.1128/AAC.00111-07

Yan, A., Guan, Z., and Raetz, C. R. (2007). An undecaprenyl phosphate-aminoarabinose flippase required for polymyxin resistance in Escherichia coli. J. Biol. Chem. 282, 36077-36089. doi: 10.1074/jbc.M7061 72200

Yao, J. D. C., and Moellering, J. R. (2011). "Antibacterial Agents," in Manual of Clinical Microbiology, 10th Edn, Vol. 1, ed. J. Versalovic (Washington: ASM Press), 1043-1081.

Zhang, L., Dhillon, P., Yan, H., Farmer, S., and Hancock, R. E. (2000). Interactions of bacterial cationic peptide antibiotics with outer and cytoplasmic membranes of Pseudomonas aeruginosa. Antimicrob. Agents Chemother. 44, 3317-3321. doi: 10.1128/AAC.44.12.3317-3321.2000 
Zhu, L., Lin, J., Ma, J., Cronan, J. E., and Wang, H. (2010). Triclosan resistance of Pseudomonas aeruginosa PAO1 is due to FabV, a triclosan-resistant enoylacyl carrier protein reductase. Antimicrob. Agents Chemother. 54, 689-698. doi: 10.1128/AAC.01152-09

Conflict of Interest Statement: The authors declare that the research was conducted in the absence of any commercial or financial relationships that could be construed as a potential conflict of interest.

Received: 15 November 2013; accepted: 24 December 2013; published online: 08 January 2014.
Citation: Morita Y, Tomida J and Kawamura Y (2014) Responses of Pseudomonas aeruginosa to antimicrobials. Front. Microbiol. 4:422. doi: 10.3389/fmicb.2013.00422 This article was submitted to Antimicrobials, Resistance and Chemotherapy, a section of the journal Frontiers in Microbiology.

Copyright (c) 2014 Morita, Tomida and Kawamura. This is an open-access article distributed under the terms of the Creative Commons Attribution License (CC BY). The use, distribution or reproduction in other forums is permitted, provided the original author(s) or licensor are credited and that the original publication in this journal is cited, in accordance with accepted academic practice. No use, distribution or reproduction is permitted which does not comply with these terms. 